\title{
Diffusiophoresis in a Suspension of Spherical Particles with Arbitrary Double-Layer Thickness
}

\author{
Yeu K. Wei and Huan J. Keh ${ }^{1}$ \\ Department of Chemical Engineering, National Taiwan University, Taipei 106-17, Taiwan, Republic of China \\ E-mail: huan@ccms.ntu.edu.tw
}

Received August 30, 2001; accepted December 10, 2001; published online February 21, 2002

\begin{abstract}
The diffusiophoresis in a homogeneous suspension of identical dielectric spheres with an arbitrary thickness of the electric double layers in a solution of a symmetrically charged electrolyte with a constant imposed concentration gradient is analytically studied. The effects of particle interactions (or particle volume fraction) are taken into account by employing a unit cell model, and the overlap of the double layers of adjacent particles is allowed. The electrokinetic equations that govern the ionic concentration distributions, the electrostatic potential profile, and the fluid flow field in the electrolyte solution surrounding the charged sphere in a unit cell are linearized assuming that the system is only slightly distorted from equilibrium. Using a perturbation method, these linearized equations are solved with the surface charge density (or zeta potential) of the particle as the small perturbation parameter. Analytical expressions for the diffusiophoretic velocity of the dielectric sphere in closed form correct to the second order of its surface charge density or zeta potential are obtained from a balance between its electrostatic and hydrodynamic forces. Comparisons of the results of the cell model with different conditions at the outer boundary of the cell are made. (1) 2002 Elsevier Science (USA)
\end{abstract}

Key Words: diffusiophoretic mobility; arbitrary double-layer thickness; effect of particle volume fraction; unit cell model.

\section{INTRODUCTION}

A colloidal particle, when placed in a solution that is not uniform in the concentration of some molecular solute, will spontaneously migrate toward regions of higher or lower concentration of the solute as a result of physical interaction between the solute molecules and the particle. The particle movement associated with this mechanism, which is called diffusiophoresis, has been analyzed extensively in the past for an isolated particle suspended in solutions of either ionic or nonionic solutes (1-8). In a solution of uncharged solute, the solute molecules interact with the particle through the van der Waals and dipole forces. For a charged particle in an electrolyte solution, the particle-solute interaction is electrostatic in nature and its range is the Debye

\footnotetext{
${ }^{1}$ To whom correspondence should be addressed. Fax: +886-2-2362-3040.
}

screening length $\kappa^{-1}$. A particle with a zeta potential of order $k T / e$ ( $\sim 25 \mathrm{mV}$; $e$ is the charge of a proton, $k$ is Boltzmann's constant, and $T$ is the absolute temperature) in an electrolyte solution with a concentration gradient of order $1 \mathrm{M} / \mathrm{cm}$ can move by diffusiophoresis at a speed of several micrometers per second.

The results for the diffusiophoretic mobility of an isolated charged particle obtained in previous studies (3-8) are valid only for a particle suspended in electrolyte solutions that extend to infinity in all directions. However, in practical applications of diffusiophoresis, colloidal particles usually are not isolated and might interact with nearby particles and/or boundaries. When the thickness of the electric double layer surrounding a particle is much smaller than the local radii of curvature of the particle such that the effect of polarization (relaxation effect) of the diffuse ions in the double layer due to nonuniform "osmotic" flow is negligible, the normalized velocity field of the immense fluid that is dragged by a particle during diffusiophoresis is the same as that for electrophoresis of the particle (5); thus, the particle interaction effects and boundary effects on electrophoresis under the situation of infinitesimally thin double layers, which have been investigated extensively in the past and summarized by a review article (9), can be utilized to interpret those on diffusiophoresis.

On the other hand, when the polarization effect of diffuse ions in the double layers of finite thickness surrounding the particles is considered, the particle-interaction behavior in diffusiophoresis can be quite different from that in electrophoresis (7). Through the use of a boundary collocation method, the diffusiophoresis of a string of dielectric spheres with thin but polarized double layers (applicable for cases with $\kappa a \geq 20$, where $a$ is the particle radius) along the line of their centers in electrolyte solutions was examined, and numerical results for the diffusiophoretic mobilities of the particles were presented for various cases (10). By employing a method of reflections, the thin-layer polarization model was also used to derive formulas for the diffusiophoretic velocities of two charged spheres with arbitrary radii, arbitrary zeta potentials, and arbitrary orientation with respect to the imposed electrolyte gradient correct to $\mathrm{O}\left(r_{12}^{-7}\right)$, where $r_{12}$ is the distance between the particle centers (11). On the basis of the concept of statistical mechanics, the reflection 
result of interaction effects between pairs of spheres with thin but polarized double layers was utilized to evaluate the average diffusiophoretic mobilities of particles in a dilute suspension subjected to a constant electrolyte gradient to the first order of the volume fraction of particles. Recently, using a unit cell model (12-17), the present authors derived analytical expressions for the diffusiophoretic mobility in a homogeneous suspension of identical dielectric spheres with thin but polarized double layers as functions of the volume fraction of particles (18). However, the effects of particle volume fraction (or particle interactions) on the diffusiophoretic mobility for a suspension of particles with relatively thick double layers have not been investigated yet.

In this work, the unit cell model is used to obtain analytical expressions for the diffusiophoretic mobility in a suspension of identical charged spheres in a solution of a symmetrically charged electrolyte with a uniform prescribed concentration gradient. No assumption is made about the thickness of the electric double layers relative to the radius of the particles, and the overlap of adjacent double layers is allowed. In the next section, we present the fundamental electrokinetic equations and boundary conditions that govern the electrolyte ion distributions, the electrostatic potential profile, and the fluid flow field for the system of a dielectric sphere in a unit cell. These basic equations are linearized assuming that the ion concentrations, the electric potential, and the fluid pressure have only a slight deviation from equilibrium due to the application of the electrolyte gradient. In Section 3, the problem of diffusiophoresis of the charged sphere in the cell containing the electrolyte solution is solved. Using the Debye-Hückel approximation, we first obtain the solution of the equilibrium electric potential distribution. The linearized electrokinetic equations are then transformed into a set of differential equations by a perturbation method with the surface charge density of the particle as the small perturbation parameter. The perturbed ion concentraion (or electrochemical potential energy), electric potential, fluid velocity, and dynamic pressure profiles are determined by solving this set of differential equations subject to the appropriate boundary conditions. Analytical expressions for the diffusiophoretic velocity of the charged sphere result from satisfying the requirement that the net force acting on the particle vanishes. Finally, closed-form expressions in a limiting case and typical numerical results of the diffusiophoretic mobility for a suspension of charged spheres are presented in Section 4. Comparisons of the previous results for diffusiophoretic particles surrounded by thin double layers (18) with our calculations are made.

\section{BASIC ELECTROKINETIC EQUATIONS}

We consider the diffusiophoretic motion of a statistically homogeneous distribution of identical charged spherical particles in a bounded solution of a symmetrically charged binary electrolyte at the steady state. The applied electrolyte concentration gradient $\nabla n^{\infty}$ is a constant equal to $\left|\nabla n^{\infty}\right| \mathbf{e}_{z}$ and the diffusiophoretic velocity of the dielectric particles is $U \mathbf{e}_{z}$, where $\mathbf{e}_{z}$ is

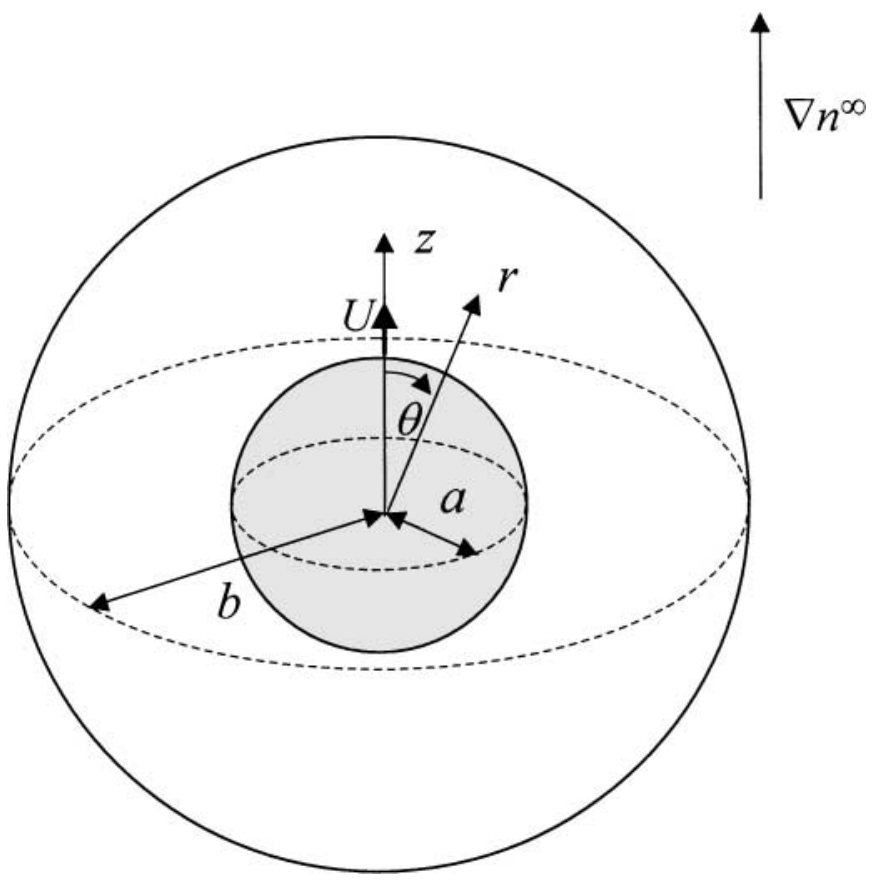

FIG. 1. Geometrical sketch for the diffusiophoresis of a spherical particle at the center of a spherical cell.

a unit vector (in the positive $z$ direction). As shown in Fig. 1, we employ a unit cell model in which each particle of radius $a$ is surrounded by a concentric spherical shell of suspending solution having an outer radius of $b$ such that the particle/cell volume ratio is equal to the particle volume fraction $\varphi$ throughout the entire suspension; viz., $\varphi=(a / b)^{3}$. The cell as a whole is electrically neutral. The origin $\mathbf{0}$ of the spherical coordinate system $(r, \theta, \phi)$ is taken at the center of the particle and the axis $\theta=0$ points toward the positive $z$ direction. Obviously, the problem for each cell is axially symmetric about the $z$ axis. The differential equations describing diffusiophoresis in electrolyte solutions are the same as those for electrophoresis. The difference is in the boundary conditions prescribed at the outer (virtual) surface of the cell.

Conservation of both ionic species requires that

$$
\nabla \cdot \mathbf{J}_{ \pm}=0
$$

where $\mathbf{J}_{ \pm}(r, \theta)$ are the number flux distributions and the subscripts + and - refer to the cation and anion, respectively. If the solution is dilute, the fluxes can be given by

$$
\mathbf{J}_{ \pm}=n_{ \pm} \mathbf{u}-D_{ \pm}\left(\nabla n_{ \pm} \pm \frac{Z e n_{ \pm}}{k T} \nabla \psi\right),
$$

where $Z$ is the valence of the symmetric electrolyte which is positive, $n_{ \pm}(r, \theta)$ and $D_{ \pm}$are respectively the concentration (number density) distributions and diffusion coefficients of the ionic species, $\mathbf{u}(r, \theta)$ is the fluid velocity field relative to the particle, and $\psi(r, \theta)$ is the electrostatic potential distribution. The 
first term on the right-hand side of Eq. [2] is the convection of the ionic species by the fluid, and the second term denotes the diffusion and electrically induced migration of the species.

We assume that the Reynolds number of the fluid motion is very small, so the inertial effect on the fluid momentum balance can be neglected. The fluid flow is governed by the Stokes equations modified with the electrostatic effect,

$$
\begin{aligned}
\eta \nabla^{2} \mathbf{u} & =\nabla p+Z e\left(n_{+}-n_{-}\right) \nabla \psi \\
\nabla \cdot \mathbf{u} & =0
\end{aligned}
$$

where $\eta$ is the viscosity of the fluid and $p(r, \theta)$ is the dynamic pressure distribution.

The local potential $\psi$ and the space charge density are related by Poisson's equation,

$$
\nabla^{2} \psi=-\frac{4 \pi}{\varepsilon} Z e\left(n_{+}-n_{-}\right)
$$

In this equation, $\varepsilon=4 \pi \varepsilon_{0} \varepsilon_{\mathrm{r}}$, where $\varepsilon_{\mathrm{r}}$ is the relative permittivity of the electrolyte solution and $\varepsilon_{0}$ is the permittivity of a vacuum. In Eqs. [2], [3], and [5], the properties $D_{ \pm}, \eta$, and $\varepsilon$ are assumed to be constant.

The boundary conditions at the surface of the dielectric particle are

$$
\begin{array}{ll}
r=a: \quad & \mathbf{u}=\mathbf{0}, \\
& \mathbf{e}_{r} \cdot \mathbf{J}_{ \pm}=0, \\
& \mathbf{e}_{r} \cdot \nabla \psi=-\frac{4 \pi}{\varepsilon} \sigma,
\end{array}
$$

where $\mathbf{e}_{r}$ is the unit normal outward from the particle surface and $\sigma$ is the uniform surface charge density of the particle. In Eq. [6a], we have assumed that the "shear plane" coincides with the particle surface. Equations [6b] and [6c] state that no ions can penetrate into the particle and the Gauss condition holds at the surface of the particle, respectively.

Because the bulk concentration of the electrolyte, $n^{\infty}$, is not uniform, it is required that the total fluxes of cations and anions are balanced in order to have no current arising from the diffusive fluxes of the electrolyte ions in an electrically neutral solution, and an electric field $\mathbf{E}^{\infty}=-\nabla \psi^{\infty}$ occurs spontaneously due to the difference in ionic mobilities. At the virtual surface of the cell, the local ionic concentration gradient and electric field are compatible with the gradient $\nabla n^{\infty}$ and induced field $\mathbf{E}^{\infty}$, respectively. Thus, the boundary conditions there are

$$
\begin{aligned}
r=b: \frac{\partial n_{ \pm}}{\partial r} & =\frac{\partial n^{\infty}}{\partial r}, \\
\frac{\partial \psi}{\partial r} & =\frac{\partial \psi^{\infty}}{\partial r}, \\
u_{r} & =-U \cos \theta,
\end{aligned}
$$

$$
\begin{aligned}
\tau_{r \theta}= & \eta\left[r \frac{\partial}{\partial r}\left(\frac{u_{\theta}}{r}\right)+\frac{1}{r} \frac{\partial u_{r}}{\partial \theta}\right]=0 \\
& (\text { for the Happel model }), \\
(\nabla \times \mathbf{u})_{\phi}= & \frac{1}{r} \frac{\partial}{\partial r}\left(r u_{\theta}\right)-\frac{1}{r} \frac{\partial u_{r}}{\partial \theta}=0
\end{aligned}
$$

(for the Kuwabara model),

where $U$ is the diffusiophoretic velocity of the particle to be determined, and $u_{r}$ and $u_{\theta}$ are the $r$ and $\theta$ components, respectively, of $\mathbf{u}$. The overlap of the adjacent double layers is allowed in Eqs. [7a] and [7b]. The Happel cell model (12) assumes that the radial velocity and the shear stress of the fluid on the outer boundary of the cell are zero, while the Kuwabara cell model (13) assumes that the radial velocity and the vorticity of the fluid are zero there. Note that the Happel model has an advantage over the Kuwabara model in that the former does not require an exchange of mechanical energy between the cell and the environment (19). Because the reference frame is taken to travel with the particle, the radial velocity given by Eq. [7c] is generated by the particle velocity in the opposite direction. The condition given by Eq. [7c] implies that there is no net flow of fluid between adjacent cells; it is valid because the suspension of the particles is bounded by impermeable walls.

To calculate the diffusiophoretic velocity of the particle in a cell, we shall assume that the prescribed electrolyte gradient is not high, and hence that the electric double layer surrounding the particle is only slightly distorted from equilibrium by the application of the gradient. Therefore, the concentration distribution of each ionic species, the electric potential distribution, and the dynamic pressure distribution have small deviations from equilibrium, and one can write

$$
\begin{aligned}
p & =p^{(\mathrm{eq})}+\delta p, \\
n_{ \pm} & =n_{ \pm}^{(\mathrm{eq})}+\delta n_{ \pm}, \\
\psi & \rightarrow \psi^{(\mathrm{eq})}+\delta \psi
\end{aligned}
$$

where $p^{(\mathrm{eq})}(r), n_{ \pm}^{(\mathrm{eq})}(r)$, and $\psi^{(\mathrm{eq})}(r)$ are the equilibrium distributions of dynamic pressure, ionic concentrations, and electric potential, respectively, and $\delta p(r, \theta), \delta n_{ \pm}(r, \theta)$, and $\delta \psi(r, \theta)$ are the small perturbations to the equilibrium state (in which no bulk concentration gradient or electric field is imposed). The equilibrium concentrations of the ions are related to the equilibrium potential by the Boltzmann distribution,

$$
n_{ \pm}^{(\mathrm{eq})}=n^{\infty}(\mathbf{0}) \exp \left(\mp \frac{Z e \psi^{(\mathrm{eq})}}{k T}\right) .
$$

The use of the bulk concentration $n^{\infty}(\mathbf{0})$ at the particle center in the above relation is valid when the solution is only slightly nonuniform in the imposed electrolyte concentration on the length scale of the particle radius $\left(\alpha=a\left|\nabla n^{\infty}\right| / n^{\infty}(\mathbf{0}) \ll 1\right)$.

Substituting Eq. [8] into Eqs. [1], [3], and [5], canceling their equilibrium components, using Eq. [9], and neglecting the 
products of the small quantities $\mathbf{u}, \delta n_{ \pm}$, and $\delta \psi$, one obtains

$$
\begin{aligned}
\nabla^{2} \delta \mu_{ \pm}= & \pm \frac{Z e}{k T}\left(\nabla \psi^{(\mathrm{eq})} \cdot \nabla \delta \mu_{ \pm}-\frac{k T}{D_{ \pm}} \nabla \psi^{(\mathrm{eq})} \cdot \mathbf{u}\right) \\
\eta \nabla^{2} \mathbf{u}= & \nabla \delta p-\frac{\varepsilon}{4 \pi}\left(\nabla^{2} \psi^{(\mathrm{eq})} \nabla \delta \psi+\nabla^{2} \delta \psi \nabla \psi^{(\mathrm{eq})}\right) \\
\nabla^{2} \delta \psi= & \frac{4 \pi Z e n^{\infty}(\mathbf{0})}{\varepsilon k T}\left[\exp \left(\frac{Z e \psi^{(\mathrm{eq})}}{k T}\right)\left(\delta \mu_{-}+Z e \delta \psi\right)\right. \\
& \left.-\exp \left(-\frac{Z e \psi^{(\mathrm{eq})}}{k T}\right)\left(\delta \mu_{+}-Z e \delta \psi\right)\right] .
\end{aligned}
$$

Here $\delta \mu_{ \pm}(r, \theta)$ is defined as a linear combination of $\delta n_{ \pm}$and $\delta \psi$ based on the concept of the electrochemical potential energy $(7,20)$,

$$
\delta \mu_{ \pm}=k T \frac{\delta n_{ \pm}}{n_{ \pm}^{(\mathrm{eq})}} \pm Z e \delta \psi
$$

The boundary conditions for $\delta \mu_{ \pm}$and $\delta \psi$ resulting from Eqs. [6] and [7] and their equilibrium state are

$$
\begin{aligned}
r=a: \quad \frac{\partial \delta \mu_{ \pm}}{\partial r} & =0, \\
\frac{\partial \delta \psi}{\partial r} & =0 \\
r=b: \quad \frac{\partial \delta \mu_{ \pm}}{\partial r} & =k T(1 \mp \beta) \frac{\alpha}{a} \cos \theta, \\
\frac{\partial \delta \psi}{\partial r} & =-\frac{k T}{Z e} \beta \frac{\alpha}{a} \cos \theta,
\end{aligned}
$$

where

$$
\beta=\frac{D_{+}-D_{-}}{D_{+}+D_{-}}
$$

Expression [15b] for the induced electric field is derived from the requirement that the ionic fluxes $\mathbf{J}_{+}$and $\mathbf{J}_{-}$defined by Eq. [2] are equal in the bulk solution $(1,3)$. The fluid velocity $\mathbf{u}$ is a small perturbed quantity, and the boundary conditions for $\mathbf{u}$ have been given by Eqs. [6a], [7c], and [7d] or [7e].

The boundary conditions of the ionic concentrations and the electric potential at the virtual surface $r=b$ may be taken as the distributions giving rise to the imposed gradient $\nabla n^{\infty}$ in the cell when the particle does not exist. In this case, Eqs. [15a] and [15b] are replaced by

$$
\begin{gathered}
r=b: \quad \delta \mu_{ \pm}=k T(1 \mp \beta) \alpha \frac{r}{a} \cos \theta, \\
\delta \psi=-\frac{k T}{Z e} \beta \alpha \frac{r}{a} \cos \theta .
\end{gathered}
$$

\section{SOLUTION FOR THE DIFFUSIOPHORETIC VELOCITY}

Before solving for the problem of diffusiophoresis of a charged sphere in a unit cell filled with the solution of a symmetric electrolyte with a constant bulk concentration gradient $\nabla n^{\infty}$, we need to determine the equilibrium electrostatic potential first. The equilibrium potential $\psi^{(\mathrm{eq})}$ satisfies the Poisson-Boltzmann equation, resulting from the substitution of the Boltzmann distribution (Eq. [9]) into Poisson's equation (Eq. [5]), the boundary condition [6c], and the requirement of no electric current passing through the virtual surface $r=b$ at equilibrium. It is easy to show that

$$
\psi^{(\mathrm{eq})}(r)=\psi_{\mathrm{eq} 1} \bar{\sigma}+\mathrm{O}\left(\bar{\sigma}^{3}\right)
$$

where $\bar{\sigma}=4 \pi Z e \sigma / \varepsilon \kappa k T$, which is the nondimensional surface charge density of the particle,

$$
\psi_{\mathrm{eq} 1}(r)=\frac{k T}{Z e}\left(\frac{\kappa a}{A}\right) \frac{a}{r}\left[(\kappa b+1) \mathrm{e}^{\kappa(a+r)}+(\kappa b-1) \mathrm{e}^{\kappa(a+2 b-r)}\right],
$$

and

$$
A=(\kappa b-1)(\kappa a+1) \mathrm{e}^{2 \kappa b}-(\kappa a-1)(\kappa b+1) \mathrm{e}^{2 \kappa a} .
$$

Here $\kappa$ is the reciprocal Debye length, defined by $\kappa=$ $\left[8 \pi Z^{2} e^{2} n^{\infty}(\mathbf{0}) / \varepsilon k T\right]^{1 / 2}$, in which $n^{\infty}(\mathbf{0})$ can be experimentally taken as the mean bulk concentration of the electrolyte in the vicinity of the diffusiophoretic particle (or in the cell). Expression [18] for $\psi^{(\mathrm{eq})}$ as a power series in the surface charge density of the particle up to $\mathrm{O}(\bar{\sigma})$ is the equilibrium solution to the linearized Poisson-Boltzmann equation, which is valid for small values of the electric potential (the Debye-Hückel approximation). That is, the surface charge density or surface potential of the particle must be small enough for the potential field $\psi^{(\mathrm{eq})}(r)$ to remain small. Note that the contribution from the effects of $\mathrm{O}\left(\bar{\sigma}^{2}\right)$ to $\psi^{(\mathrm{eq})}$ disappears only for the case of symmetric electrolytes.

Using Eqs. [18]-[20], one obtains a relation between the surface potential and the surface charge density of the dielectric sphere in a unit cell at equilibrium,

$$
\bar{\sigma}=W \frac{Z e \zeta}{k T},
$$

where

$$
W=\frac{\gamma \cosh \gamma+\left(\kappa^{2} a^{2}+\kappa a \gamma-1\right) \sinh \gamma}{\kappa a[(\kappa a+\gamma) \cosh \gamma-\sinh \gamma]},
$$

$\zeta=\psi^{(\mathrm{eq})}(a)$, which is the equilibrium surface potential (known as the zeta potential) of the particle, and $\gamma=\kappa a\left(\varphi^{-1 / 3}-1\right)$. Namely, the solution for $\psi^{(\mathrm{eq})}(r)$ given by Eq. [18] with the substitution of Eq. [21] is also valid for a dielectric sphere with 
a constant surface potential in the cell. In the limiting case of $\varphi=0$, Eq. [22] reduces to the simple formula $W=1+(\kappa a)^{-1}$ for an isolated charged sphere.

To solve the small quantities $\delta \mu_{ \pm}, \mathbf{u}, \delta p$, and $\delta \psi$ in terms of the particle velocity $U$ when the parameter $\bar{\sigma}$ is small, these variables can be written as perturbation expansions in powers of $\bar{\sigma}$,

$$
\begin{aligned}
\delta \mu_{ \pm} & =\mu_{0 \pm}+\mu_{1 \pm} \bar{\sigma}+\mu_{2 \pm} \bar{\sigma}^{2}+\cdots, \\
\mathbf{u} & =\mathbf{u}_{1} \bar{\sigma}+\mathbf{u}_{2} \bar{\sigma}^{2}+\cdots, \\
\delta p & =p_{1} \bar{\sigma}+p_{2} \bar{\sigma}^{2}+\cdots, \\
\delta \psi & =\psi_{0}+\psi_{1} \bar{\sigma}+\psi_{2} \bar{\sigma}^{2}+\cdots, \\
U & =U_{1} \bar{\sigma}+U_{2} \bar{\sigma}^{2}+\cdots,
\end{aligned}
$$

where the functions $\mu_{i \pm}, \mathbf{u}_{i}, p_{i}, \psi_{i}$, and $U_{i}$ are independent of $\bar{\sigma}$. The zeroth-order terms of $\mathbf{u}, \delta p$, and $U$ disappear because an uncharged particle will not move by imposing an electrolyte concentration gradient if only the electrostatic interaction is considered.

Substituting the expansions given by Eq. [23] and $\psi^{(\mathrm{eq})}$ given by Eq. [18] into the governing Eqs. [4] and [10]-[12] and boundary conditions [6a], [7c], [7d] or [7e], [14], and [15] or [17], and equating like powers of $\bar{\sigma}$ on both sides of the respective equations, we obtain a group of linear differential equations and boundary conditions for each set of the functions $\mu_{i \pm}, \mathbf{u}_{i}, p_{i}$, and $\psi_{i}$ with $i$ equal to 0,1 , and 2 . These perturbation equations can be analytically solved, and the results for the $r$ and $\theta$ components of $\mathbf{u}, \delta p$ (to the order of $\bar{\sigma}^{2}$ ), $\delta \mu_{ \pm}$, and $\delta \psi$ (to the order of $\bar{\sigma}$ ) can be written as

$$
\begin{aligned}
u_{r}= & \left\{\left[U_{1} F_{0 r}(r)-\frac{k T}{\eta a^{2}} \beta \alpha F_{1 r}(r)\right] \bar{\sigma}\right. \\
& \left.+\left[U_{2} F_{0 r}(r)+\frac{k T}{\eta a^{2}} \alpha F_{2 r}(r)\right] \bar{\sigma}^{2}\right\} \cos \theta \\
u_{\theta}= & \left\{\left[U_{1} F_{0 \theta}(r)-\frac{k T}{\eta a^{2}} \beta \alpha F_{1 \theta}(r)\right] \bar{\sigma}\right. \\
& \left.+\left[U_{2} F_{0 \theta}(r)+\frac{k T}{\eta a^{2}} \alpha F_{2 \theta}(r)\right] \bar{\sigma}^{2}\right\} \sin \theta \\
\delta p= & \frac{\eta}{a}\left\{\left[U_{1} F_{p 0}(r)-\frac{k T}{\eta a^{2}} \beta \alpha F_{p 1}(r)-\frac{\varepsilon \kappa^{2} a k T}{4 \pi \eta Z e} \beta \alpha \psi_{\mathrm{eq} 1}(r)\right.\right. \\
& \left.\times F_{\psi 0}(r)\right] \bar{\sigma}+\left[U_{2} F_{p 0}(r)+\frac{k T}{\eta a^{2}} \alpha F_{p 2}(r)\right. \\
& \left.\left.+\frac{\varepsilon \kappa^{2} a k T}{4 \pi \eta Z e} \alpha \psi_{\mathrm{eq} 1}(r) F_{\psi 1}(r)\right] \bar{\sigma}^{2}\right\} \cos \theta \\
\delta \mu_{ \pm}= & k T(1 \mp \beta) \alpha\left[F_{\mu 0}(r) \mp F_{\mu 1}(r) \bar{\sigma}\right] \cos \theta \\
\delta \psi= & \frac{k T}{Z e} \alpha\left[-\beta F_{\psi 0}(r)+F_{\psi 1}(r) \bar{\sigma}\right] \cos \theta
\end{aligned}
$$

Here $F_{i r}(r), F_{i \theta}(r), F_{p i}(r)$ (with $i$ equal to 0,1 , and 2$), F_{\mu 0}(r)$, $F_{\mu 1}(r), F_{\psi 0}(r)$, and $F_{\psi 1}(r)$ are dimensionless functions of $r$ given by Eqs. [A1] and [A6]-[A8] in the Appendix. Note that the solutions for $\delta \mu_{ \pm}$and $\delta \psi$ to the order of $\bar{\sigma}$ do not contain the influence of the fluid motion.

The total force exerted on the charged sphere undergoing diffusiophoresis in a unit cell filled with an electrolyte solution can be expressed as the sum of the electric force and the hydrodynamic drag force. Since the net charge within a cell is zero, the electric force acting on the charged sphere can be represented by the integral of the electrostatic force density over the fluid volume in the cell. Because the net electric force acting on the particle at the equilibrium state is zero, the leading order of the electric force is given by

$\mathbf{F}_{\mathrm{e}}=-\frac{\varepsilon}{2} \int_{0}^{\pi} \int_{a}^{b}\left(\nabla^{2} \psi^{(\mathrm{eq})} \nabla \delta \psi+\nabla^{2} \delta \psi \nabla \psi^{(\mathrm{eq})}\right) r^{2} \sin \theta d r d \theta$.

Substituting Eqs. [18] and [26] into Eq. [27] and performing the integration, we obtain

$$
\begin{aligned}
\mathbf{F}_{\mathrm{e}}= & \frac{k T}{a} \alpha\left\{-\beta\left[\frac{\varepsilon \kappa^{2} a^{3}}{3 Z e} \psi_{\mathrm{eq} 1}(a) F_{\psi 0}(a)-\frac{\varepsilon \kappa^{2} a b^{2}}{3 Z e} \psi_{\mathrm{eq} 1}(b) F_{\psi 0}(b)\right.\right. \\
& \left.+4 \pi \int_{a}^{b}\left(\frac{r}{a}\right)^{3} G_{1}(r) d r\right] \bar{\sigma}+\left[\frac{\varepsilon \kappa^{2} a^{3}}{3 Z e} \psi_{\mathrm{eq} 1}(a) F_{\psi 1}(a)\right. \\
& -\frac{\varepsilon \kappa^{2} a b^{2}}{3 Z e} \psi_{\mathrm{eq} 1}(b) F_{\psi 1}(b) \\
& \left.\left.+4 \pi \int_{a}^{b}\left(\frac{r}{a}\right)^{3} G_{2}(r) d r\right] \bar{\sigma}^{2}+\mathrm{O}\left(\bar{\sigma}^{3}\right)\right\} \mathbf{e}_{z}
\end{aligned}
$$

where

$$
\begin{aligned}
G_{1}(r) & =\frac{\varepsilon \kappa^{2} a^{4}}{12 \pi Z e r} F_{\mu 0}(r) \frac{d \psi_{\mathrm{eq} 1}}{d r} \\
G_{2}(r) & =-\frac{\varepsilon \kappa^{2} a^{4}}{12 \pi Z e r}\left[F_{\mu 1}(r)+\frac{Z e}{k T} \psi_{\mathrm{eq} 1}(r) F_{\mu 0}(r)\right] \frac{d \psi_{\mathrm{eq} 1}}{d r}
\end{aligned}
$$

In Eqs. [28] and [29], the function $\psi_{\text {eq1 }}(r)$ has been given by Eq. [19], and $F_{\mu i}(r)$ and $F_{\psi i}(r)$ with $i$ equal to 0 and 1 are defined by Eqs. [A6]-[A8].

The hydrodynamic drag force acting on the colloidal sphere is given by the integral of the hydrodynamic stress over the particle surface,

$$
\mathbf{F}_{\mathrm{h}}=2 \pi a^{2} \int_{0}^{\pi}\left\{-\delta p \mathbf{e}_{r}+\eta\left[\nabla \mathbf{u}+(\nabla \mathbf{u})^{\mathrm{T}}\right] \cdot \mathbf{e}_{r}\right\}_{r=a} \sin \theta d \theta .
$$


Substitution of Eq. [24] into the above equation results in

$$
\begin{aligned}
\mathbf{F}_{\mathrm{h}}= & -\left\{\left[4 \pi \eta a U_{1} C_{02}-4 \pi \frac{k T}{a} \beta \alpha C_{12}\right.\right. \\
& \left.-\frac{\varepsilon \kappa^{2} a^{2} k T}{3 Z e} \beta \alpha \psi_{\mathrm{eq} 1}(a) F_{\psi 0}(a)\right] \bar{\sigma} \\
& +\left[4 \pi \eta a U_{2} C_{02}+4 \pi \frac{k T}{a} \alpha C_{22}\right. \\
& \left.\left.+\frac{\varepsilon \kappa^{2} a^{2} k T}{3 Z e} \alpha \psi_{\mathrm{eq} 1}(a) F_{\psi 1}(a)\right] \bar{\sigma}^{2}+\mathrm{O}\left(\bar{\sigma}^{3}\right)\right\} \mathbf{e}_{z} .
\end{aligned}
$$

For the case of a dielectric sphere with a constant (equilibrium) surface potential in the cell, the parameter $\bar{\sigma}$ in Eqs. [23]-[26], [28], and [31] can be replaced by Ze $/ k T$ in terms of Eq. [21].

At the steady state, the total force acting on the diffusiophoretic particle is zero. Applying this constraint to the summation of Eqs. [28] and [31], one obtains

$$
\begin{aligned}
U_{1} & =\frac{\varepsilon \beta \alpha}{4 \pi \eta a}\left(\frac{k T}{Z e}\right)^{2} \frac{\Theta_{1}}{W}, \\
U_{2} & =\frac{\varepsilon \alpha}{32 \pi \eta a}\left(\frac{k T}{Z e}\right)^{2} \frac{\Theta_{2}}{W^{2}},
\end{aligned}
$$

where $W$ is defined by Eq. [22], and $\Theta_{i}$ with $i$ equal to 1 and 2 are functions of $\kappa a$ and $\varphi$ defined by

$$
\begin{aligned}
\Theta_{i}= & \frac{(-8)^{i-1}}{3\left(3+2 \varphi^{5 / 3}\right)}(\kappa a)^{2} W^{i} \frac{Z e}{k T}\left\{\frac{2}{\varphi^{2 / 3} \omega} \psi_{\mathrm{eq} 1}(b) F_{\psi(i-1)}(b)\right. \\
& -\frac{12 \pi Z e}{\varepsilon \kappa^{2} a^{3}} \int_{a}^{b}\left[1-3 \frac{r^{2}}{a^{2}}+2 \frac{r^{3}}{a^{3}}-\varphi^{5 / 3}\right. \\
& \left.\left.\times\left(2 \frac{r^{2}}{a^{2}}-3 \frac{r^{3}}{a^{3}}+\frac{r^{5}}{a^{5}}\right)\right] G_{i}(r) d r\right\}
\end{aligned}
$$

for the Happel model, and

$$
\begin{aligned}
\Theta_{i}= & \frac{(-8)^{i-1}}{9}(\kappa a)^{2} W^{i} \frac{Z e}{k T}\left\{\frac{2}{\varphi^{2 / 3} \omega^{\prime}} \psi_{\mathrm{eq} 1}(b) F_{\psi(i-1)}(b)\right. \\
& -\frac{12 \pi Z e}{\varepsilon \kappa^{2} a^{3}} \int_{a}^{b}\left[1-3 \frac{r^{2}}{a^{2}}+2 \frac{r^{3}}{a^{3}}-\frac{1}{5} \varphi\right. \\
& \left.\left.\times\left(2-5 \frac{r^{3}}{a^{3}}+3 \frac{r^{5}}{a^{5}}\right)\right] G_{i}(r) d r\right\}
\end{aligned}
$$

for the Kuwabara model. In the above equations,

$$
\begin{aligned}
\omega & =\left(1-\frac{3}{2} \varphi^{1 / 3}+\frac{3}{2} \varphi^{5 / 3}-\varphi^{2}\right)^{-1}, \\
\omega^{\prime} & =\left(1-\frac{9}{5} \varphi^{1 / 3}+\varphi-\frac{1}{5} \varphi^{2}\right)^{-1},
\end{aligned}
$$

From Eqs. [23e] and [32], the diffusiophoretic velocity of the charged sphere in the cell can be expressed as

$$
U=\frac{\varepsilon \alpha}{4 \pi \eta a W}\left(\frac{k T}{Z e}\right)^{2}\left[\beta \Theta_{1} \bar{\sigma}+\frac{1}{8 W} \Theta_{2} \bar{\sigma}^{2}+\mathrm{O}\left(\bar{\sigma}^{3}\right)\right] .
$$

Since the solutions for $\delta \mu_{ \pm}$and $\delta \psi$ given by Eqs. [25] and [26] are not influenced by the fluid flow, the effect of the polarization (or relaxation) of the diffuse ions in the electric double layer surrounding the particle is not included in Eq. [35] up to the order $\bar{\sigma}^{2}$. Substitution of Eq. [21] into Eq. [35] yields an expression for the diffusiophoretic velocity of the particle as an expansion in powers of $\zeta$,

$$
U=\frac{\varepsilon \alpha}{4 \pi \eta a}\left[\frac{k T}{Z e} \beta \Theta_{1} \zeta+\frac{1}{8} \Theta_{2} \zeta^{2}+\mathrm{O}\left(\zeta^{3}\right)\right]
$$

Note that the diffusiophoretic mobility of dielectric spheres with a constant (equilibrium) surface potential $\zeta$ is the same as that of the spheres with a constant surface charge density $\sigma=W \varepsilon \kappa \zeta / 4 \pi$, and it can be expressed by either Eq. [35] or Eq. [36].

\section{RESULTS AND DISCUSSION}

It is understood that the diffusiophoresis of a charged particle results from a linear combination of two effects: (i) chemiphoresis due to the nonuniform adsorption of counterions in the electric double layer over the particle surface, which is analogous to the diffusiophoresis in nonionic media; (ii) electrophoresis due to the macroscopic electric field generated by the concentration gradient of the electrolyte and the difference in mobilities of the cation and anion of the electrolyte, given by Eq. [15b] or [17b]. The terms in Eqs. [35] and [36] proportional to $\beta$ (involving the function $\Theta_{1}$ ) represent the contribution from electrophoresis, while the remainder terms (containing the function $\Theta_{2}$ ) are the chemiphoretic component. Using the boundary condition [15b] for the electric potential at the virtual surface of a unit cell, one can find that the function $\Theta_{1}$ given by Eq. [33b] for the Kuwabara model is identical to that obtained by Levine and Neale (14) for the electrophoretic mobility of a dielectic sphere in the cell.

In the limit of a very dilute suspension $(\varphi \rightarrow 0)$, Eq. [33] becomes

$$
\begin{aligned}
\Theta_{1}= & 1-\mathrm{e}^{\kappa a}\left[5 E_{7}(\kappa a)-2 E_{5}(\kappa a)\right], \\
\Theta_{2}= & 1+\frac{1}{3} \mathrm{e}^{2 \kappa a}\left[10 E_{6}(2 \kappa a)+7 E_{8}(2 \kappa a)\right]-40 \mathrm{e}^{2 \kappa a} E_{7}(\kappa a) \\
& \times\left[E_{3}(\kappa a)-E_{5}(\kappa a)\right]+\frac{4}{3} \mathrm{e}^{\kappa a}\left[3 E_{3}(\kappa a)\right. \\
& \left.+9 E_{4}(\kappa a)-7 E_{5}(\kappa a)-15 E_{6}(\kappa a)\right],
\end{aligned}
$$


where

$$
E_{n}(\kappa a)=(\kappa a)^{n-1} \int_{\kappa a}^{\infty} t^{-n} \mathrm{e}^{-t} d t .
$$

The formulas for $\Theta_{1}$ and $\Theta_{2}$ in Eq. [37] are the reduced results obtained earlier for the diffusiophoresis of an isolated dielectric sphere (8). Note that $\Theta_{1}$ given by Eq. [37a] is the same as that derived by Henry (21) for the electrophoretic mobility of the particle.

In Figs. 2 and 3, the numerical results for the function $\Theta_{1}$ calculated from Eq. [33] are plotted versus the parameters $\kappa a$
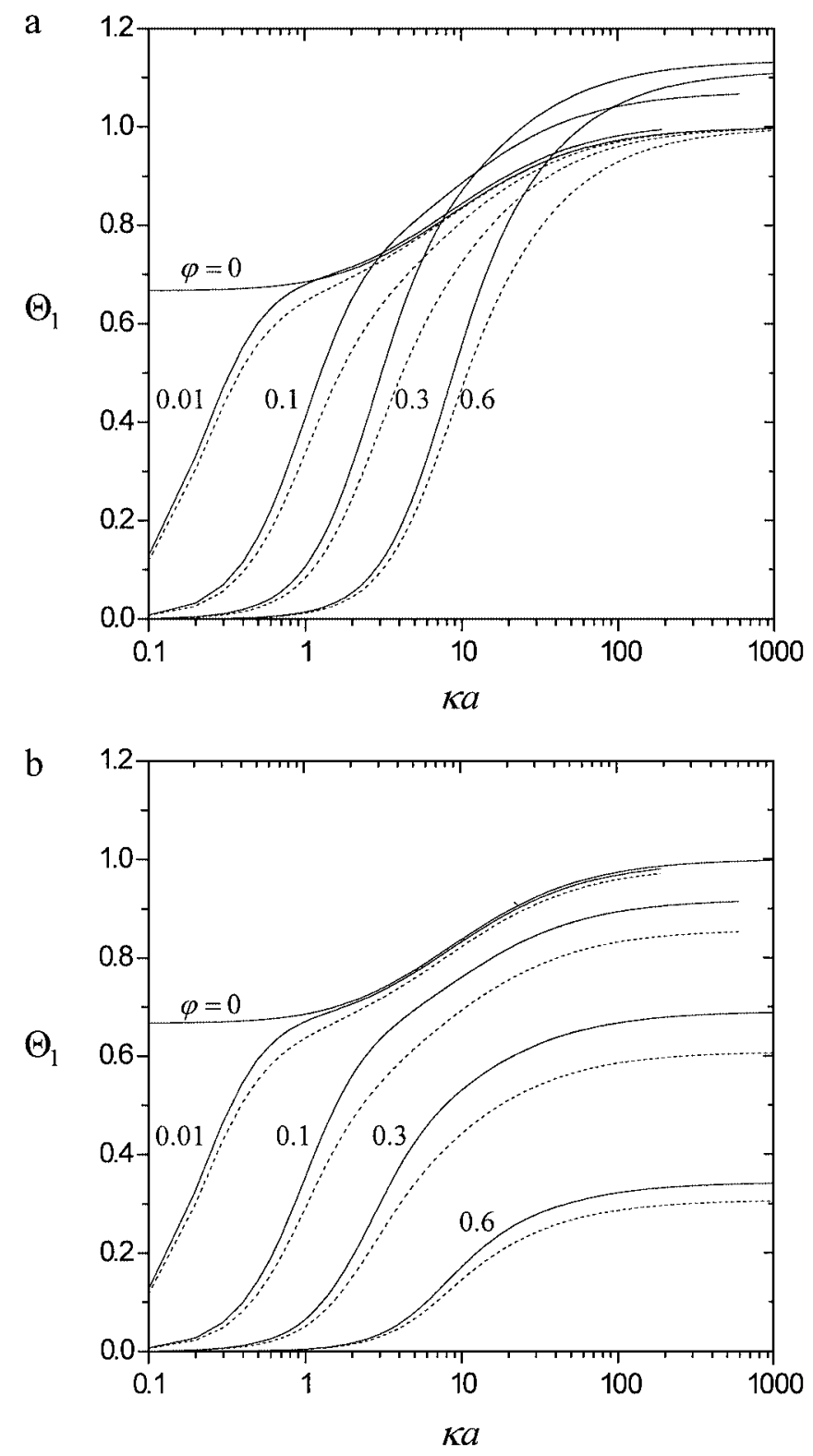

FIG. 2. Plots of the function $\Theta_{1}$ as calculated from Eq. [33] versus $\kappa a$ with $\varphi$ as a parameter: (a) when boundary condition [15] is used, and (b) when boundary condition [17] is used. The solid and dashed curves represent the calculations for the Happel and Kuwabara models, respectively.
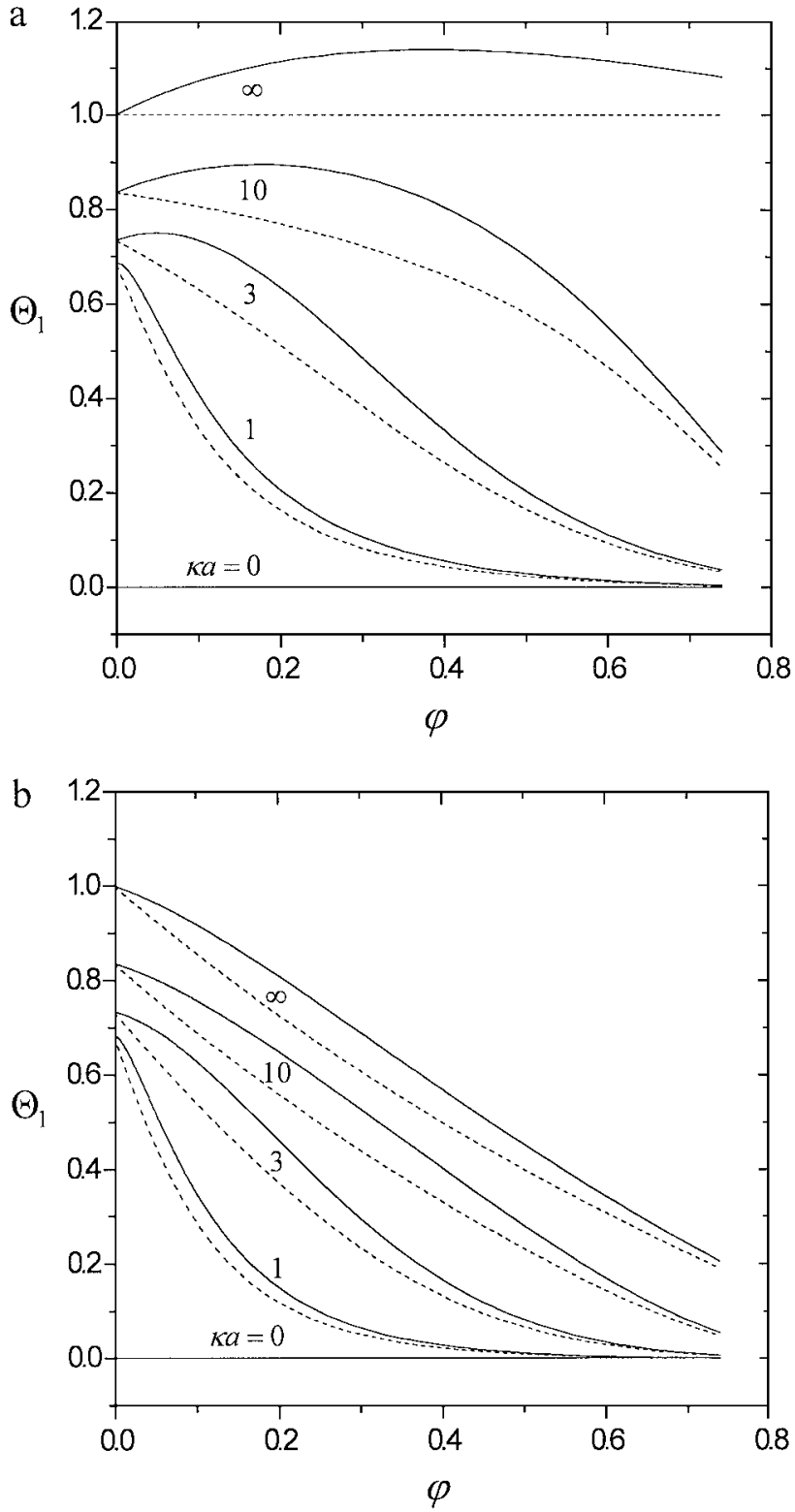

FIG. 3. Plots of the function $\Theta_{1}$ as calculated from Eq. [33] versus $\varphi$ with $\kappa a$ as a parameter: (a) when boundary condition [15] is used, and (b) when boundary condition [17] is used. The solid and dashed curves represent the calculations for the Happel and Kuwabara models, respectively.

and $\varphi$ for the unit cell model with various boundary conditions at the virtual surface of the cell. The calculations are presented up to $\varphi=0.74$, which corresponds to the maximum attainable volume fraction for a swarm of identical spheres (14). It can be seen that $\Theta_{1}$ is always positive and decreases monotonically with the decrease of $\kappa a$ (or with the increase of the double-layer overlap) for a specified value of $\varphi$. When $\kappa a=0, \Theta_{1}=2 / 3$ as $\varphi=0$, and $\Theta_{1}=0$ for all finite values of $\varphi$. If the double layers are relatively thick $(\kappa a<1), \Theta_{1}$ is always a monotonic decreasing function of $\varphi$ for a fixed value of $\kappa a$, and the particles' volume fraction effect on the electrophoretic mobility is significant even 
in fairly dilute suspensions. When the boundary condition [15] for the electrochemical potential at the virtual surface of the cell is used, $\Theta_{1}$ is a monotonic decreasing function of $\varphi$ for any given finite value of $\kappa a$ (and equals unity as $\kappa a \rightarrow \infty$, regardless of the value of $\varphi$ ) for the case of the Kuwabara model. However, for the case of the Happel model, $\Theta_{1}$ is not a monotonic function of $\varphi$ and has a maximum (whose value can be greater than 1) for a given value of $\kappa a$ higher than about unity. The location of this maximum shifts to greater $\varphi$ as $\kappa a$ increases. On the other hand, when the boundary condition [17] for the electrochemical potential at the outer surface of the cell is used, both the Happel and the Kuwabara models predict that $\Theta_{1}$ decreases monotonically with an increase in $\varphi$ for an arbitrary fixed value of $\kappa a$. For any combination of $\kappa a$ and $\varphi$, the Kuwabara model results in a somewhat lower value of $\Theta_{1}$ (or a stronger particles' volume fraction dependence for the electrophoretic mobility) than the Happel model does. This occurs because the zero-vorticity boundary condition yields a energy dissipation in the cell larger than that due to particle drag alone, owing to the additional work done by the stresses at the virtual surface (19). On the other hand, the value of $\Theta_{1}$ calculated using the boundary condition [17] is always smaller than that calculated using the boundary condition [15].

The results for the function $\Theta_{2}$ calculated from Eq. [33] are plotted versus the parameters $\kappa a$ and $\varphi$ in Figs. 4 and 5 for various cases of the cell model. It can be seen that $\Theta_{2}$ in general is not a monotonic function of $\varphi$ for a specified value of $\kappa a$. When the boundary condition [15] is used, $\Theta_{2}$ is not a monotonic function of $\kappa a$ for a given value of $\varphi$, and a local maximum and a minimum of this function would appear at some values of $\kappa a$. The value of $\Theta_{2}$ is negative in the vicinity of each minimum and positive otherwise. For relatively concentrated suspensions, the value of $\Theta_{2}$ in the vicinity of each maximum may be greater than unity. For a combination of $\kappa a$ and $\varphi$ not close to these minima, the Kuwabara model predicts a somewhat smaller value of $\Theta_{2}$ than the Happel model does, similar to their predictions for the value of $\Theta_{1}$. On the other hand, when the boundary condition [17] is used, the value of $\Theta_{2}$ is always positive and in general increases with an increase in $\kappa a$ for a constant value of $\varphi$. When the value of $\kappa a$ is large, $\Theta_{2}$ decrease monotonically with an increase in $\varphi$ from the maximum value of unity at $\varphi=0$. For any combination of $\kappa a$ and $\varphi$, the Kuwabara model again leads to a slightly lower value of $\Theta_{2}$ than the Happel model does.

Figure 6 illustrates the dependence of the diffusiophoretic velocity for a suspension of identical charged spheres on their dimensionless surface (zeta) potential at various values of $\kappa a$ and $\varphi$ calculated for the Kuwabara model with boundary condition [17]. The Kuwabara model with boundary condition [17] is chosen because the diffusiophoretic mobility predicted by this case of the unit cell model agrees well with the ensembleaveraged result obtained by using the concept of statistical mechanics for a dilute suspension of particles with thin but polarized double layers (18). The consequence that the boundary condition [15] is not as accurate as the boundary condition
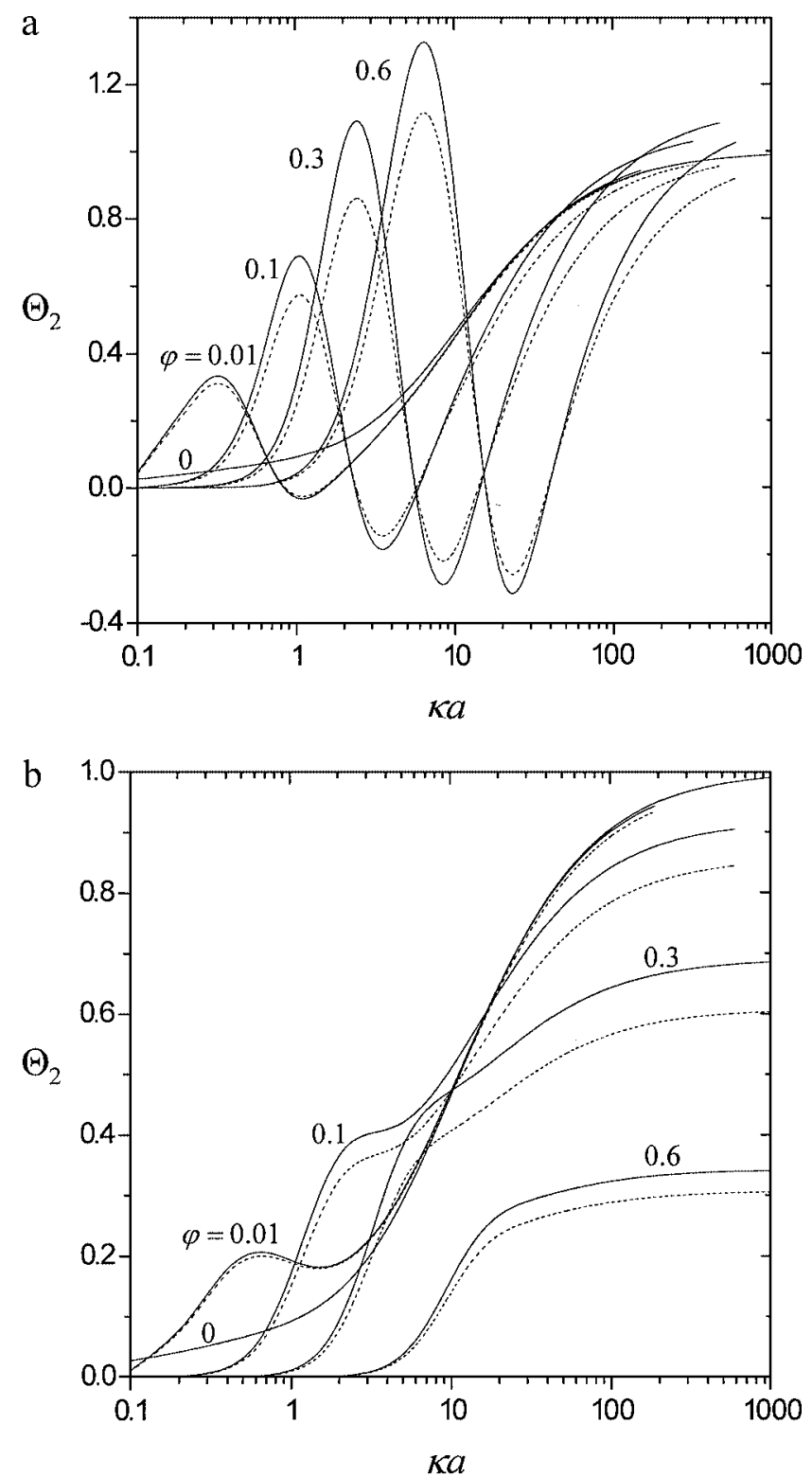

FIG. 4. Plots of the function $\Theta_{2}$ as calculated from Eq. [33] versus $\kappa a$ with $\varphi$ as a parameter: (a) when boundary condition [15] is used, and (b) when boundary condition [17] is used. The solid and dashed curves represent the calculations for the Happel and Kuwabara models, respectively.

[17] is probably due to the fact that the angular component of the electrochemical/electrostatic potential gradients at the virtual surface of the cell is not specified in Eq. [15]. A possible reason for the outcome that the Kuwabara model is more appropriate than the Happel model for this suspension might be the fact that the zero-vorticity boundary condition is consistent with the irrotational-flow environment generated by a diffusiophoretic particle with a thin polarized double layer. Note that, however, except for the case with $\kappa a \rightarrow \infty$, the flow caused by two or more identical diffusiophoretic spheres is not irrotational (11). 
a

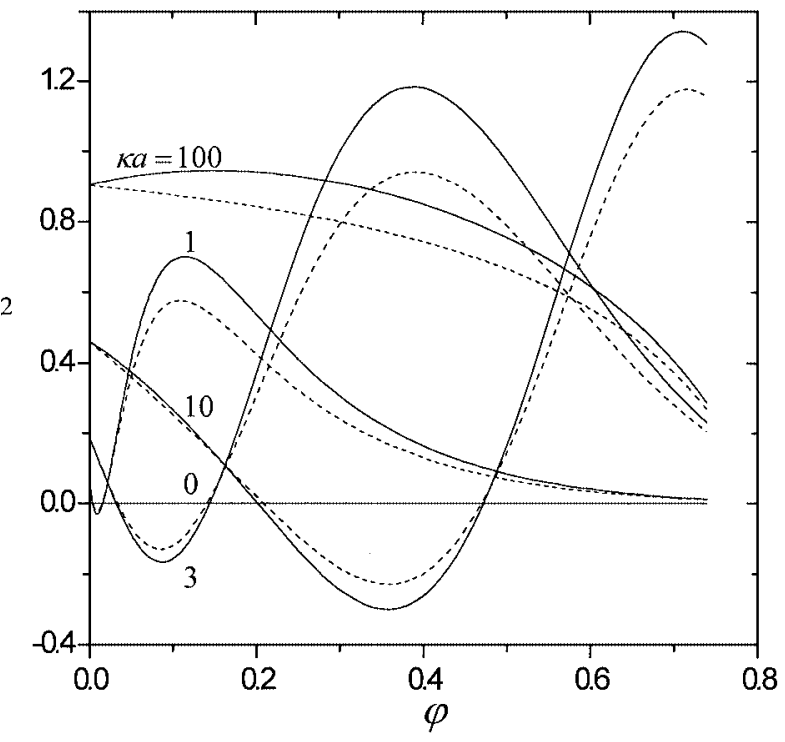

b

$\Theta_{2}$

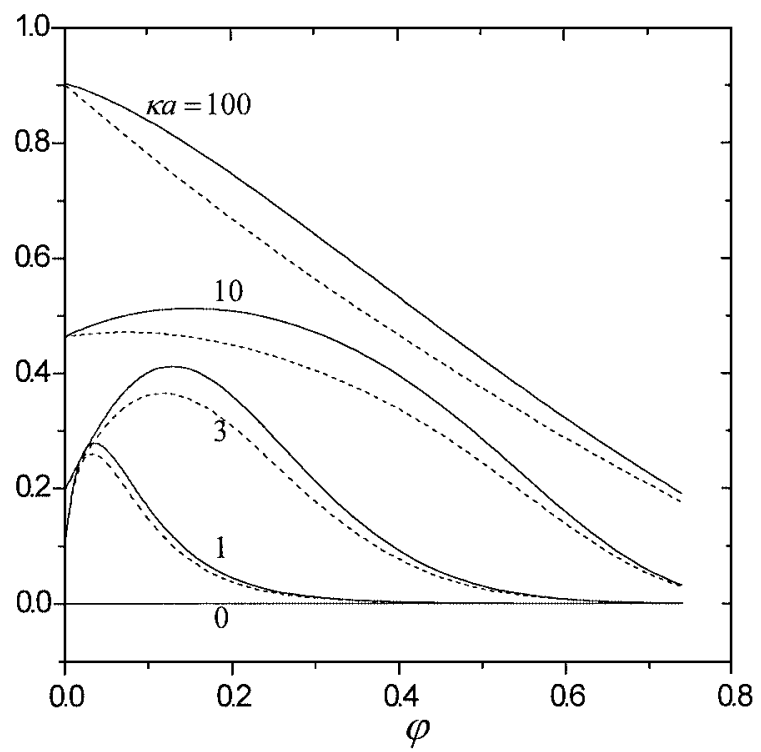

FIG. 5. Plots of the function $\Theta_{2}$ as calculated from Eq. [33] versus $\varphi$ with $\kappa a$ as a parameter: (a) when boundary condition [15] is used, and (b) when boundary condition [17] is used. The solid and dashed curves represent the calculations for the Happel and Kuwabara models, respectively.

The magnitude of the diffusiophoretic velocity shown in Fig. 6 is normalized by a characteristic value given by

$$
U^{*}=\frac{\varepsilon \alpha}{4 \pi \eta a}\left(\frac{k T}{Z e}\right)^{2} .
$$

The case that the anion and cation diffusivities are equal $(\beta=0)$ is drawn in Fig. 6a. Only the results at positive surface potentials are shown because the diffusiophoretic velocity, which is due to the chemiphoretic effect entirely, is an even function of the surface potential $\zeta$ as indicated by Eq. [36]. Since our analysis is based on the assumption of small surface potential, the magnitudes of $Z e \zeta / k T$ considered are less than 2. As expected, in this range of $Z e \zeta / k T$, the magnitude of the reduced diffusiophoretic velocity $U / U^{*}$ increases monotonically with an increase in $Z e \zeta / k T$ for given values of $\kappa a$ and $\varphi$. There is no chemiphoretic motion of the particles for the special cases of $Z e \zeta / k T=0$ or $\kappa a=0$. Figure $6 \mathrm{~b}$ is plotted for the reduced diffusiophoretic mobility $U / U^{*}$ of the particles for a case that
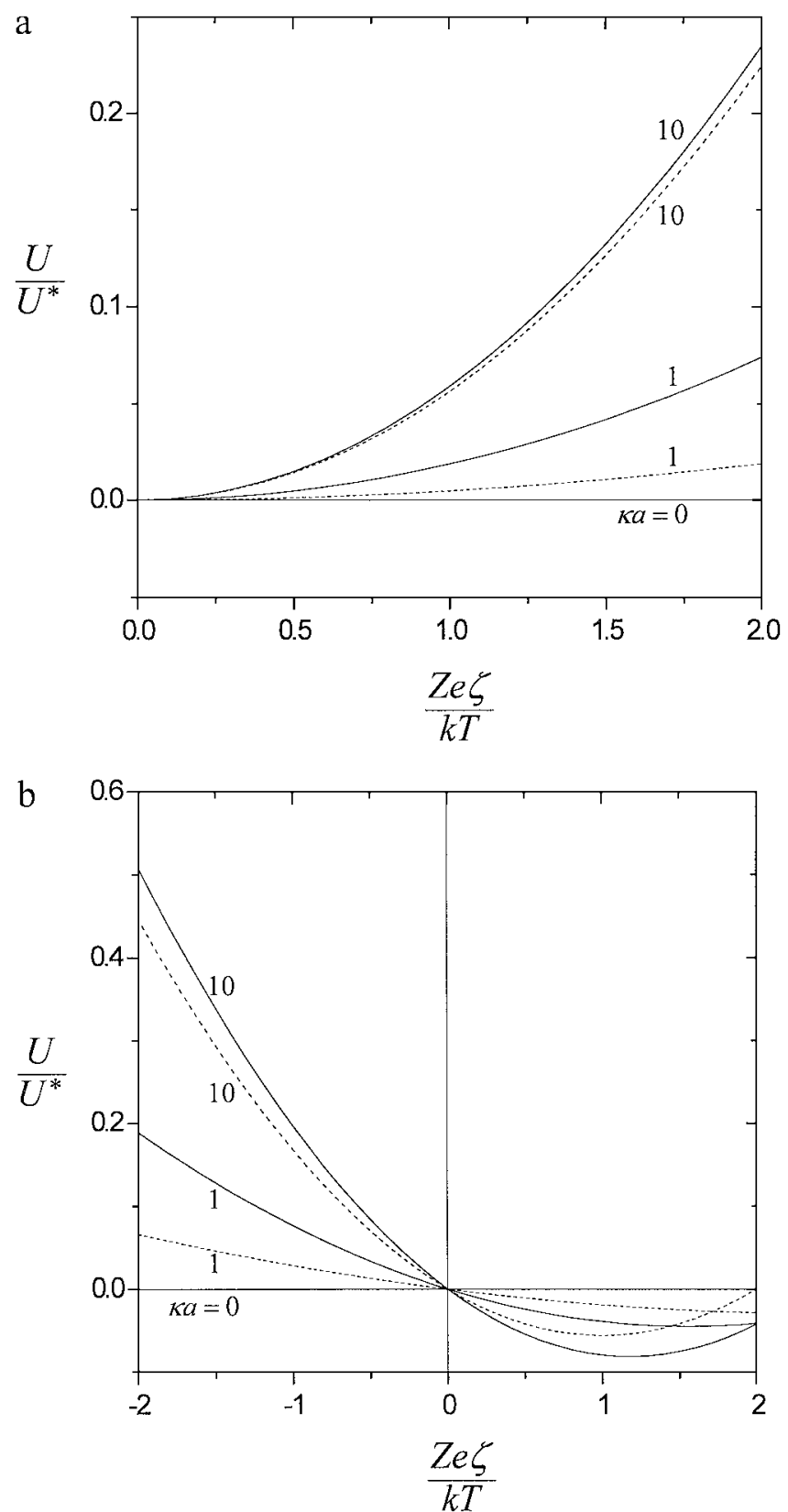

FIG. 6. Plots of the reduced diffusiophoretic mobility in a suspension of identical spheres versus the dimensionless zeta potential at fixed values of $\kappa a$ calculated for the Kuwabara model with boundary condition [17]: (a) $\beta=0$, and (b) $\beta=-0.2$. The solid and dashed curves represent the cases of $\varphi=0.1$ and $\varphi=0.2$, respectively. 

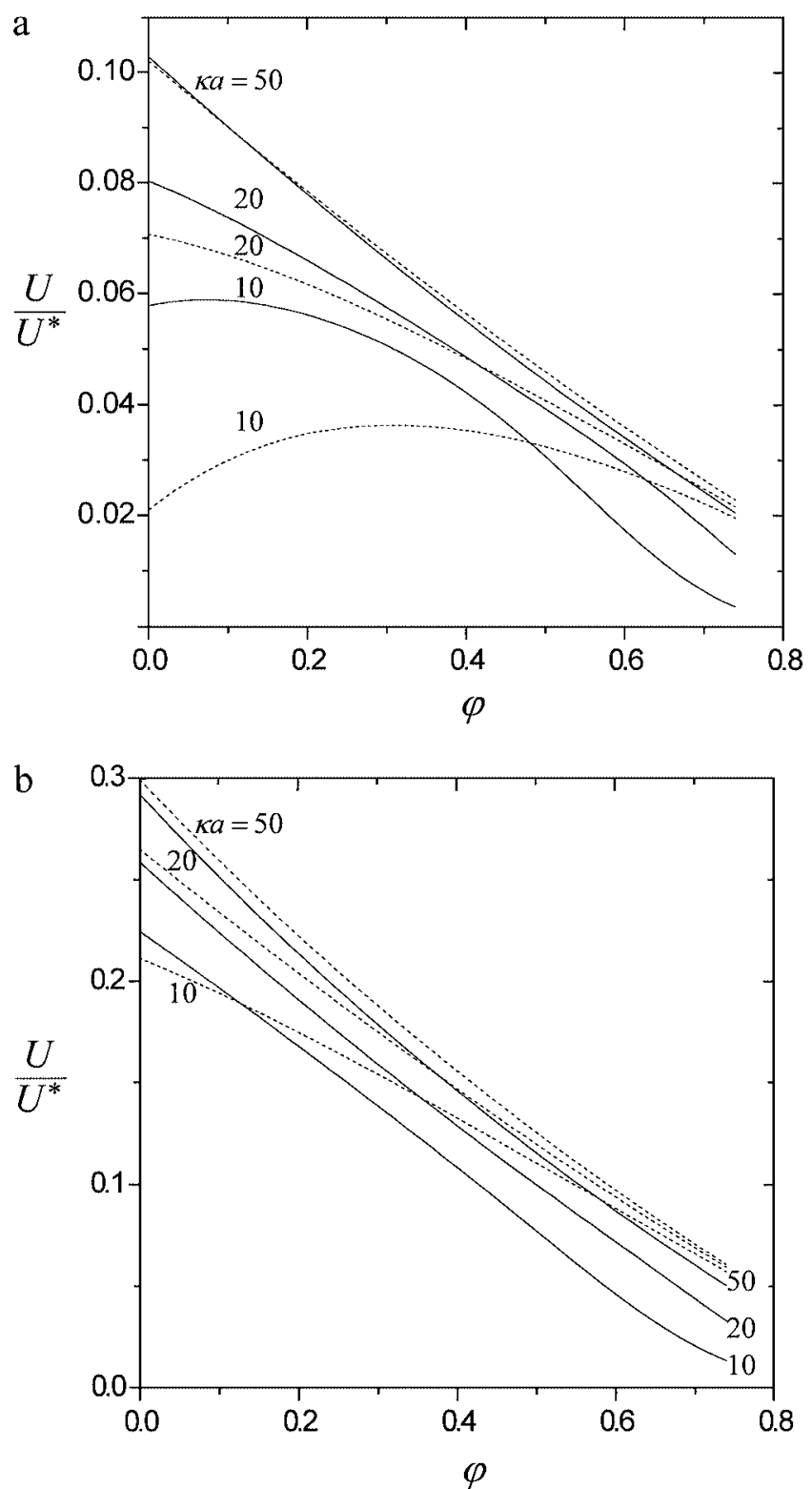

FIG. 7. Plots of the reduced diffusiophoretic mobility in a suspension of identical spheres versus $\varphi$ at $Z e \zeta / k T=-1$ and several values of $\kappa a$ calculated for the Kuwabara model with boundary condition [17]: (a) $\beta=0$, and (b) $\beta=-0.2$. The solid and dashed curves represent our present results and those obtained by using the thin-layer polarization model (18), respectively.

the cation and anion of the electrolyte have different diffusion coefficients $(\beta=-0.2$ is chosen). In this case, both the chemiphoretic and the electrophoretic effects contribute to the particles' movement and the net diffusiophoretic velocity is neither an even nor an odd function of the surface potential. It can be seen that $U / U^{*}$ is not necessarily a monotonic function of $Z e \zeta / k T$ given constant values of $\kappa a$ and $\varphi$. Some of the curves in Fig. $6 \mathrm{~b}$ show that the particles might reverse direction of movement more than once as their surface potential varies from negative to positive values. The reversals occur- ring at the values of $Z e \zeta / k T$ other than zero result from the competition between the contributions from chemiphoresis and electrophoresis. In the limit $\kappa a=0$, the diffusiophoretic velocity vanishes for any finite value of $\varphi$, but reduces to the Hückel result for electrophoresis $\left[U / U^{*}=(2 / 3) \beta(Z e \zeta / k T)\right]$ when the suspension is infinitely dilute (with $\varphi=0$ ). Note that the situations associated with Figs. $6 \mathrm{a}$ and $6 \mathrm{~b}$ (taking $Z=1$ ) are close to the diffusiophoresis in the aqueous solutions of $\mathrm{KCl}$ and $\mathrm{NaCl}$, respectively.

In the previous cell-model analysis for the diffusiophoretic mobility in suspensions of charged particles, the assumption of thin but polarized double layers was made (18). It would be of interest to know the extent that this assumption is valid. A comparison of the results of the reduced diffusiophoretic mobility obtained in this previous analysis with our present calculations for the Kuwabara model with boundary condition [17] is shown in Fig. 7. For the case of $Z e \zeta / k T=-1$ chosen in this figure, the polarization effect of diffuse ions in the double layers is negligible. It can be seen that the results of $U / U^{*}$ predicted by the thin-double-layer analysis can be in significant errors when the value of $\kappa a$ is less than about 20 .

\section{SUMMARY}

In this paper, the steady-state diffusiophoresis in a homogeneous suspension of identical charged spheres with an arbitrary value of $\kappa a$ in a solution of a symmetric electrolyte with a uniform imposed concentration gradient is analyzed using the unit cell model with various boundary conditions at the virtual surface of the cell. Solving the linearized continuity equations of electrolyte ions, Poisson-Boltzmann equation, and modified Stokes equations applicable to the system of a sphere in a unit cell by a regular perturbation method, we have obtained the ion concentration (or electrochemical potential energy) distributions, the electric potential profile, and the fluid flow field. The requirement that the total force exerted on the particle is zero leads to Eqs. [33]-[36] for the diffusiophoretic velocity of the charged sphere as functions of the volume fraction of the particles correct to the order $\sigma^{2}$ or $\zeta^{2}$.

Equations [33]-[36] are obtained on the basis of the DebyeHückel approximation for the equilibrium potential distribution around the dielectric sphere with low zeta potential in a unit cell. The reduced formula of Eq. [33] for $\Theta_{1}$ of a single dielectric sphere with low zeta potential in an unbounded electrolyte solution, Eq. [37a], was shown to give a good approximation for the case of reasonably high zeta potential (with errors less than $4 \%$ for $|\zeta| e / k T \leq 2)(22)$. Also, comparing with the numerical solution for the diffusiophoretic mobility of an isolated charged sphere in $\mathrm{KCl}$ and $\mathrm{NaCl}$ aqueous solutions obtained by Prieve and Roman (4) valid for an arbitrary value of zeta potential, one can find that Eq. [36] with Eq. [37] for a dilute suspension of charged spheres with low zeta potential is also quite accurate for the entire range of $|\zeta| e / k T \leq 2(8)$. Therefore, 
our results in Eqs. [33]-[36] might be used tentatively for the situation of reasonably high electric potentials. In order to see whether our approximate solution can be extended to the higher values of electric potential, a numerical solution of the electrokinetic equations with no assumption on the magnitude of electric potential would be needed to compare it with the approximate solution.

\section{APPENDIX}

\section{Definitions of Some Functions in Section 3}

For conciseness the definitions of some functions in Section 3 are listed here. In Eq. [24],

$$
\begin{aligned}
& F_{i r}(r)= C_{i 1}+C_{i 2}\left(\frac{a}{r}\right)+C_{i 3}\left(\frac{a}{r}\right)^{3}+C_{i 4}\left(\frac{r}{a}\right)^{2}+\left(\delta_{i 1}+\delta_{i 2}\right) \\
& \times\left[\frac{1}{5}\left(\frac{a}{r}\right)^{3} \int_{a}^{r}\left(\frac{r}{a}\right)^{5} G_{i}(r) d r-\frac{a}{r} \int_{a}^{r}\left(\frac{r}{a}\right)^{3} G_{i}(r) d r\right. \\
&\left.+\int_{a}^{r}\left(\frac{r}{a}\right)^{2} G_{i}(r) d r-\frac{1}{5}\left(\frac{r}{a}\right)^{2} \int_{a}^{r} G_{i}(r) d r\right], \quad[\mathrm{A} 1 \mathrm{a}] \\
& F_{i \theta}(r)=-C_{i 1}-\frac{C_{i 2}}{2}\left(\frac{a}{r}\right)+\frac{C_{i 3}}{2}\left(\frac{a}{r}\right)^{3}-2 C_{i 4}\left(\frac{r}{a}\right)^{2} \\
&+\left(\delta_{i 1}+\delta_{i 2}\right)\left[\frac{1}{10}\left(\frac{a}{r}\right)^{3} \int_{a}^{r}\left(\frac{r}{a}\right)^{5} G_{i}(r) d r+\frac{a}{2 r}\right. \\
& \times \int_{a}^{r}\left(\frac{r}{a}\right)^{3} G_{i}(r) d r-\int_{a}^{r}\left(\frac{r}{a}\right)^{2} G_{i}(r) d r \\
&\left.+\frac{2}{5}\left(\frac{r}{a}\right)^{2} \int_{a}^{r} G_{i}(r) d r\right], \\
&\left.-\left(\frac{a}{r}\right)^{2} \int_{a}^{r}\left(\frac{r}{a}\right)^{3} G_{i}(r) d r\right], \\
& F_{p i}(r)= C_{i 2}\left(\frac{a}{r}\right)^{2}+10 C_{i 4} \frac{r}{a}-\left(\delta_{i 1}+\delta_{i 2}\right)\left[\frac{2 r}{a} \int_{a}^{r} G_{i}(r) d r\right. \\
& {[\mathrm{A} 1 \mathrm{c}] }
\end{aligned}
$$

for $i=0,1$, and 2 . In the above equations, the functions $G_{i}(r)$ are defined by Eq. [29], $\delta_{i j}$ is the Kronecker delta which equals unity if $i=j$ but vanishes otherwise,

$$
\begin{aligned}
& C_{i 1}=-\frac{1}{2}\left[\left(2+3 \varphi^{5 / 3}\right) A_{i}+5 \varphi B_{i}\right] \omega, \\
& C_{i 2}=\frac{1}{2}\left[\left(3+2 \varphi^{5 / 3}\right) A_{i}+5 \varphi^{2 / 3} B_{i}\right] \omega, \\
& C_{i 3}=-\frac{1}{2}\left[A_{i}+\left(3 \varphi^{2 / 3}-2 \varphi\right) B_{i}\right] \omega, \\
& C_{i 4}=\frac{1}{2}\left[\varphi^{5 / 3} A_{i}-\left(2 \varphi^{2 / 3}-3 \varphi\right) B_{i}\right] \omega
\end{aligned}
$$

for the Happel model, and

$$
\begin{aligned}
& C_{i 1}=-\frac{1}{2}\left[(2+\varphi) A_{i}^{\prime}+\left(2-5 \varphi+3 \varphi^{5 / 3}\right) B_{i}^{\prime}\right] \omega^{\prime}, \\
& C_{i 2}=\frac{1}{2}\left[3 A_{i}^{\prime}+\left(3-5 \varphi^{2 / 3}+2 \varphi^{5 / 3}\right) B_{i}^{\prime}\right] \omega^{\prime}, \\
& C_{i 3}=-\frac{1}{10}\left[(5-2 \varphi) A_{i}^{\prime}+5\left(1-3 \varphi^{2 / 3}+2 \varphi\right) B_{i}^{\prime}\right] \omega^{\prime}, \\
& C_{i 4}=\frac{1}{10}\left[3 \varphi A_{i}^{\prime}+5\left(2 \varphi^{2 / 3}-3 \varphi+\varphi^{5 / 3}\right) B_{i}^{\prime}\right] \omega^{\prime}
\end{aligned}
$$

for the Kuwabara model, where

$$
\begin{aligned}
A_{i}= & \delta_{i 0}+\left(\delta_{i 1}+\delta_{i 2}\right)\left[\int_{a}^{b}\left(\frac{r}{a}\right)^{2} G_{i}(r) d r\right. \\
& \left.-\frac{a}{b} \int_{a}^{b}\left(\frac{r}{a}\right)^{3} G_{i}(r) d r\right], \\
B_{i}= & \left(\delta_{i 1}+\delta_{i 2}\right) \frac{1}{5}\left[\left(\frac{a}{b}\right)^{3} \int_{a}^{b}\left(\frac{r}{a}\right)^{5} G_{i}(r) d r\right. \\
& \left.-\left(\frac{b}{a}\right)^{2} \int_{a}^{b}\left(\frac{r}{a}\right)^{3} G_{i}(r) d r\right] \\
A_{i}^{\prime}= & \delta_{i 0}+\left(\delta_{i 1}+\delta_{i 2}\right)\left[\int_{a}^{b}\left(\frac{r}{a}\right)^{2} G_{i}(r) d r-\frac{a}{b} \int_{a}^{b}\left(\frac{r}{a}\right)^{3}\right. \\
& \times G_{i}(r) d r+\frac{1}{5}\left(\frac{a}{b}\right)^{3} \int_{a}^{b}\left(\frac{r}{a}\right)^{5} G_{i}(r) d r \\
& \left.-\frac{1}{5}\left(\frac{b}{a}\right)^{2} \int_{a}^{b}\left(\frac{r}{a}\right)^{3} G_{i}(r) d r\right] \\
B_{i}^{\prime}= & \left(\delta_{i 1}+\delta_{i 2}\right) \frac{1}{5}\left[-\left(\frac{a}{b}\right) \int_{a}^{b}\left(\frac{r}{a}\right)^{3} G_{i}(r) d r\right. \\
& \left.+\left(\frac{b}{a}\right)^{2} \int_{a}^{b} G_{i}(r) d r\right],
\end{aligned}
$$

and $\omega$ and $\omega^{\prime}$ are defined by Eq. [34].

In Eqs. [25] and [26],

$$
\begin{aligned}
F_{\mu 0}(r)= & F_{\psi 0}(r)=\frac{1}{\chi}\left(\frac{r}{a}+\frac{a^{2}}{2 r^{2}}\right), \\
F_{\mu 1}(r)= & \frac{Z e r}{3 k T a \chi}\left\{\frac{a^{3} A_{\mu 1}(a, b)+2(1-\chi) B_{\mu 1}(a, b)}{2 \chi r^{3}}\right. \\
& +\frac{1-\chi}{\chi}\left[A_{\mu 1}(a, b)+\frac{2}{b^{3} \varphi} B_{\mu 1}(a, b)\right] \\
& \left.+\frac{1}{r^{3}} B_{\mu 1}(a, r)+A_{\mu 1}(r, b)\right\},
\end{aligned}
$$




$$
\begin{aligned}
& F_{\psi 1}(r)=\frac{1}{\kappa^{3} r^{2}} \\
& \times\left\{\frac{\left[g_{1+}(\kappa a)-\mathrm{e}^{2 \kappa a} g_{1-}(\kappa a)\right] A_{\psi 1}(a, b)-2 g_{1+}(\kappa a) B_{\psi 1}(a, b)}{\mathrm{e}^{2 \kappa a} g_{2+}(\kappa b) g_{1-}(\kappa a)-\mathrm{e}^{2 \kappa b} g_{2-}(\kappa b) g_{1+}(\kappa a)}\right. \\
& \times\left(-g_{2+}(\kappa b)[\kappa r \cosh (\kappa r)-\sinh (\kappa r)]+\frac{1}{2}\left[g_{2+}(\kappa b)\right.\right. \\
& \left.\left.-\mathrm{e}^{2 \kappa b} \mathrm{~g}_{2-}(\kappa b)\right](\kappa r+1) \mathrm{e}^{-\kappa r}\right)-[\kappa r \cosh (\kappa r) \\
& \left.-\sinh (\kappa r)] A_{\psi 1}(r, b)+(\kappa r+1) \mathrm{e}^{-\kappa r} B_{\psi 1}(r, b)\right\},
\end{aligned}
$$

where

$$
\begin{array}{ll}
A_{\mu 1}(x, y)=\int_{x}^{y}\left(1-\frac{a^{3}}{r^{3}}\right) \frac{d \psi_{\mathrm{eq} 1}}{d r} d r, & \text { [A9a] } \\
B_{\mu 1}(x, y)=\int_{x}^{y}\left(r^{3}-a^{3}\right) \frac{d \psi_{\mathrm{eq} 1}}{d r} d r, & \text { [A9b] } \\
A_{\psi 1}(x, y)=\int_{x}^{y} \mathrm{e}^{-\kappa r} \kappa^{2}(\kappa r+1)\left[F_{\mu 1}(r)+\frac{Z e}{k T} \psi_{\mathrm{eq} 1}(r) F_{\mu 0}(r)\right] d r
\end{array}
$$

$$
\begin{aligned}
B_{\psi 1}(x, y)= & \int_{x}^{y} \kappa^{2}[\kappa r \cosh (\kappa r)-\sinh (\kappa r)] \\
& \times\left[F_{\mu 1}(r)+\frac{Z e}{k T} \psi_{\mathrm{eq} 1}(r) F_{\mu 0}(r)\right] d r, \quad \text { [A10b] }
\end{aligned}
$$$$
g_{1 \pm}(x)=2 \pm 2 x+x^{2}
$$

$g_{2 \pm}(x)=g_{1 \pm}(x)$ and $\quad \chi=1-\varphi \quad$ (if Eq. [15] is used),

[A12a,b]

$$
g_{2 \pm}(x)=1 \pm x \quad \text { and } \quad \chi=1+\varphi / 2 \quad \text { (if Eq. [17] is used). }
$$

[A12c,d]

\section{ACKNOWLEDGMENT}

This research was supported by the National Science Council of the Republic of China under Grant NSC89-2214-E-002-051.

\section{REFERENCES}

1. Dukhin, S. S., and Derjaguin, B. V., in "Surface and Colloid Science" (E. Matijevic, Ed.), Vol. 7, Wiley, New York, 1974.

2. Anderson, J. L., Lowell, M. E., and Prieve, D. C., J. Fluid Mech. 117, 107 (1982).

3. Prieve, D. C., Anderson, J. L., Ebel, J. P., and Lowell, M. E., J. Fluid Mech. 148, 247 (1984).

4. Prieve, D. C., and Roman, R., J. Chem. Soc. Faraday Trans. 2 83, 1287 (1987).

5. Anderson, J. L., Annu. Rev. Fluid Mech. 21, 61 (1989).

6. Pawar, Y., Solomentsev, Y. E., and Anderson, J. L., J. Colloid Interface Sci. 155, 488 (1993).

7. Keh, H. J., and Chen, S. B., Langmuir 9, 1142 (1993).

8. Keh, H. J., and Wei, Y. K., Langmuir 16, 5289 (2000).

9. Chen, S. B., and Keh, H. J., in "Interfacial Forces and Fields" (J. Hsu, Ed.), Dekker, New York, 1999.

10. Keh, H. J., and Luo, S. C., Langmuir 12, 657 (1996).

11. Tu, H. J., and Keh, H. J., J. Colloid Interface Sci. 231, 265 (2000).

12. Happel, J., AIChE J. 4, 197 (1958).

13. Kuwabara, S., J. Phys. Soc. Jpn. 14, 527 (1959).

14. Levine, S., and Neale, G. H., J. Colloid Interface Sci. 47, 520 (1974).

15. Zharkikh, N. I., and Shilov, V. N., Colloid J. USSR (English transl.) 43, 865 (1982).

16. Kozak, M. W., and Davis, E. J., J. Colloid Interface Sci. 129, 166 (1989).

17. Ohshima, H., J. Colloid Interface Sci. 225, 233 (2000).

18. Wei, Y. K., and Keh, H. J., Langmuir 17, 1437 (2001).

19. Happel, J., and Brenner, H., "Low Reynolds Number Hydrodynamics." Nijhoff, The Netherlands, 1983.

20. Ohshima, H., Healy, T. W., and White, L. R., J. Chem. Soc. Faraday Trans. 2 79, 1613 (1983).

21. Henry, D. C., Proc. R. Soc. London, A 133, 106 (1931).

22. O'Brien, R. W., and White, L. R., J. Chem. Soc. Faraday Trans. 2 74, 1607 (1978). 